\title{
Successful Treatment with Proton Beam Therapy Alone for Postoperative Esophageal Squamous Cell Carcinoma with Liver and Lymph Node Metastases After Failure of First-Line Chemotherapy
}

\section{Yojiro Ishikawa ( $\nabla$ y02614111@yahoo.co.jp )}

Tohoku University Hospital: Tohoku Daigaku Byoin https://orcid.org/0000-0002-4613-0332

\section{Motohisa Suzuki}

Southern Tohoku Proton Therapy Center

Hisashi Yamaguchi

Southern Tohoku Proton therapy Center Ichiro Seto

Southern Tohoku Proton Therapy Center

Masanori Machida

Southern Tohoku Proton Therapy Center

\section{Yoshiaki Takagawa}

Southern Tohoku Proton Therapy Center

Keiichi Jingu

Tohoku University: Tohoku Daigaku

Yasuyuki Kikuchi

Southern Tohoku Proton Therapy Center

Masao Murakami

Southern Tohoku Proton Therapy Center

\section{Case report}

Keywords: Esophageal cancer, Proton Beam therapy, Liver metastasis, lymph node metastasis

Posted Date: October 18th, 2021

DOI: https://doi.org/10.21203/rs.3.rs-960347/v1

License: (c) (1) This work is licensed under a Creative Commons Attribution 4.0 International License.

Read Full License 


\section{Abstract}

Background: Cisplatin (CDDP) and 5-fluorouracil (5-FU) are used in first-line chemotherapy for recurrent esophageal squamous cell carcinoma (ESCC) after radical esophagectomy; however, patients have a poor prognosis after failure of first-line treatment. We herein report the achievement of long-term survival after proton therapy in an ESCC patient in whom the first-line chemotherapy failed.

Case presentation: A 60-year-old man was diagnosed as having squamous cell carcinoma of the lower thoracic esophagus (CT2NOMO, Stage IIA). He underwent two courses of neoadjuvant chemotherapy with $\mathrm{CDDP}$ and 5-FU, and esophagectomy with three-field lymphadenectomy was performed. Microscopic findings after resection showed two lymph node metastases (ypT2N1M0, Stage IIB). Five months after surgery, a computed tomography scan revealed a solitary liver metastasis in the S4 area. He underwent three courses of chemotherapy with CDDP and 5-FU. Although surgeons decided to perform resection for the liver metastasis, preoperative positron emission tomography (PET) revealed two lymph node metastases. Surgeons recommended second-line chemotherapy or chemoradiotherapy, bud the patient refused second-line chemotherapy and requested proton beam therapy (PBT). We performed PBT without chemotherapy for the liver metastasis and lymph node metastases with total dose of 79.2 and $60 \mathrm{~Gy}$ relative biological effectiveness, respectively, according to the tumor location. The PBT resulted in a complete response as shown by PET. An acute side effect of grade 1 dermatitis occurred after PBT, but there was no acute or late complication of more than grade 2. The patient remains in complete remission 5 years after treatment without surgery or chemotherapy.

Conclusions: PBT exerted a curative effect on postoperative recurrence of ESCC. This is the first report about PBT for postoperative ESCC with liver and lymph node metastases after failure of first-line chemotherapy for metastases in distant organs.

\section{Background}

Esophageal squamous cell carcinoma (ESCC) is one of the most difficult malignancies to cure because of its early metastasis. Radical esophagectomy has improved the survival of patients with ESCC, but the rate of recurrence is high (27-52\%)(1-4). Cisplatin (CDDP) and 5-fluorouracil (5-FU) are used in first-line chemotherapy for recurrent esophageal cancer after radical esophagectomy; however, patients have a poor prognosis after failure of first-line treatment (5).

Proton beam therapy (PBT) is effective because protons have excellent dose localization according to the Bragg peak compared with photons and are biologically equivalent to conventional X-ray treatment for cancer(6-9). We herein report the achievement of long-term survival after proton therapy in an ESCC patient in whom the first-line chemotherapy for postoperative solitary liver metastasis and two lymph node metastases failed.

\section{Case Presentation}


One year before presentation to our hospital, a male Japanese patient was diagnosed as having squamous cell carcinoma (SCC) of the lower thoracic esophagus (cT2NOM0, Stage IIA) at 60 years of age. He underwent two courses of neoadjuvant chemotherapy with CDDP in addition to 5-FU, and esophagectomy with three-field lymphadenectomy followed by gastric tube reconstruction for SCC of the lower thoracic esophagus was performed. Microscopic findings after resection showed that the tumor was moderately differentiated SCC. Additionally, two lymph node metastases were observed (ypT2N1M0, Stage IIB).

Five months after surgery, a CT scan revealed a solitary liver metastasis measuring $21 \times 15 \mathrm{~mm}$ in size in the S4 area (Fig. 1). He underwent three courses of chemotherapy with CDDP in addition to 5-FU. During the three courses chemotherapy, the patient suffered from side effects of chemotherapy including nausea and loss of appetite. Follow-up PET after the three courses of chemotherapy reveled two new lymph node metastases in the para-aorta area (Fig. 2). The surgeons judged that the first-line chemotherapy had failed because the lymph node metastases showed an increase in size.

The surgeons therefore recommended resection of the liver metastasis with retroperitoneal lymph node dissection. However, the patient refused to receive surgery due to concerns about the treatment outcome and side effects despite the fact that the patient had an Eastern Cooperative Oncology Group Performance Score of 0 . The surgeons also recommended chemoradiotherapy or second-line chemotherapy. However, the patient refused to undergo second-line chemotherapy or chemoradiotherapy due to concerns about the treatment side effects.

He requested alternative treatment and was referred to our institution. We considered stereotactic radiotherapy or radio-frequency ablation for the liver metastasis. However, we did not select these therapies because he had two lymph node metastases outside the liver.

We therefore recommended the combination of PBT (or RT) and chemotherapy. The patient did not accept the RT and chemotherapy, and he requested PBT without chemotherapy.

We explained the risks and benefits of PBT alone to the patient. One problem with PBT is the high cost of treatment, but the patient was covered by personal medical insurance for PBT. Finally, we decided to perform PBT alone for the liver metastasis and lymph node metastases.

The PBT system at our institute (Proton beam system, Mitsubishi, Tokyo, Japan) uses synchrotron and scattering methods. Treatment planning for PBT was based on three-dimensional CT images taken at 2$\mathrm{mm}$ intervals in the exhalation phase while using a respiratory gating system (Anzai Medical, Tokyo, Japan). Treatment was administered during the exhalation phase using a respiratory gating system. Daily front and lateral $\mathrm{X}$-ray imaging was used for positioning.

The gross tumor volume (GTV) included the liver and lymph node metastases. The clinical target volume (CTV) was defined as GTV plus $0.5-\mathrm{cm}$ margins. The planning target volume (PTV) was CTV plus $0.5-\mathrm{cm}$ margins. 
We decided to reduce the daily dose for the lymph node metastases because they were located close to the stomach. The daily PBT fractions were 3.3 and 2.0 Gy relative biological effectiveness (RBE) for liver metastasis and lymph node metastases, respectively. The liver metastasis and lymph node metastases received total doses of 79.2 and 60 Gy RBE, respectively (Fig. 3).

An acute side effect of grade 1 dermatitis according to the National Cancer Institute Common Terminology Criteria for Adverse Events version 4.0. occurred after PBT, but there was no acute or late complication of more than grade 2. PET after treatment showed no evidence of recurrence (Fig. 4). The patient remains in complete remission 5 years after treatment without surgery or chemotherapy.

\section{Discussion And Conclusions}

A total irradiation dose of 60-70 Gy has been used for radiation therapy for recurrent ESCC without chemotherapy, and the five-year overall survival rate was reported to be $11 \%-22 \%$ (11). Murori et al. also reported that primary tumors including mediastinal lymph nodes and liver metastases received proton beam irradiation at a total dose of 66-68 Gy RBE with chemotherapy(12). In addition, it was reported that high-dose (72.0-90.8 Gy RBE) proton beam therapy with or without chemotherapy was an efficacious and safe treatment for patients with $\operatorname{ESCC}(13)$.

The present case was treated with PBT for multiple recurrence of ESCC without chemotherapy. It is not surprising that abdominal lymph node metastases from various cancers are well controlled by PBT without chemotherapy; however, there have been few reports on successful treatment with PBT without chemotherapy for distant metastases from ESCC.

Chemotherapy with CDDP and 5-FU is the first-line treatment for unresectable advanced cancer and recurrent ESCC. However, second-line treatment after failure of chemotherapy with CDDP and 5-FU is controversial. Treatment with a single agent such as paclitaxel, docetaxel, navelvine, erlotinib or irinotecan and some combination therapies have been performed. Previous studies showed response rates of $12.5 \%-44.2 \%$ and median survival periods of 6-10.4 months. On the other hand, the median survival period of patients with ESCC for whom first-line therapy failed is only 3 to 6 months if they do not receive chemotherapy(14-19). Despite the fact that first-line therapy in our patient with ESCC failed, we were able to control liver metastasis and lymph node metastases because of the high dose of PBT and the biological effective advantage of PBT compared to conventional X-ray therapy.

It has been reported that some patients with malignancies may refuse standard treatment. According to a retrospective review of the Surveillance Epidemiology and End Results (SEER) database between 2004 and 2013 , the rate of surgery refusal was $0.64 \%$. It was also suggested in that review that age, ethnicity, marital status, disease stage, and lack of insurance are associated with higher risk of refusal of surgery(10). Considering report, it is notable that our unique point was that we were able to use PBT for the patient despite the fact that the patient had refused standard therapy. 
Cancer status with less than five metastatic or recurrent lesions and with controlled primary lesions can be considered as "oligo-recurrence"(20). A previous study showed that oligo-recurrence in lymph nodes of patients with esophageal cancer can be cured by chemoradiotherapy(21). Little is known about the survival of patients with specific subtypes of recurrent disease after radical esophagectomy. Patients with a small number of lymph node metastases and patients with metastasis in a solid organ such as the liver, brain or lung from esophageal cancer have been reported to have a significantly longer median survival period $(21,22)$. Therefore, the prognosis may not be poor even if a small number of lymph node metastases are combined with isolated liver metastases. However, there has been no study on the outcome of treatment when a small number of lymph node metastases in a single solid organ occur at the same time. Generally, patients with organ metastases and lymph node metastases have a poor prognosis. Kato et al. reported a classification of recurrent ESCC after radical esophagectomy with a longer survival period in cases of locoregional recurrence than in cases of distant or mixed recurrence and a shorter survival period in cases of locoregional recurrence in more than one lymph node station and in a solid organ such as the bone, pleura, or peritoneum (22).

The abscopal effect is probably associated with our good local control without chemotherapy for recurrence of ESCC by PBT. This effect is known as an immune effect caused by high doses of radiation and other factors. The biological mechanism underlying this effect remains unclear. There have been few case reports on an abscopal effect for esophageal cancer. Zhao $X$ et al. reported an abscopal effect of radiation on lymph node metastasis in an ESCC patient who received Cyberknife radiotherapy with a dose of $42 \mathrm{~Gy}$ in 6 daily fractions(23). To the best of our knowledge, there has been no study on an abscopal effect of PBT for ESCC.

This report is an interesting case report on "off-label" management of a single non-colorectal nonneuroendocrine liver metastasis with lymph nodal disease progression in the para-aortic area. It is reasonable to consider that our case was oligo-recurrence of a solitary organ and localized lymph node metastases with good prognosis. Because this study is was case study, it is difficult to define the indication for salvage therapy for postoperative recurrence of esophageal cancer. However, it is possible that some patients with single solid organ metastasis with localized lymph node metastases were treated only by chemotherapy despite being potential candidates of PBT.

PBT for recurrent ESCC and metastatic lesions is therefore considered to be an effective therapeutic option. We hope that multicenter trials will be carried out and that indications will be expanded.

\section{Abbreviations}

ESCC: Esophageal squamous cell carcinoma

CDDP: Cisplatin

CT: Computed tomography 
CTV: Clinical target volume

GTV: Gross tumor volume

Gy: Gray

PBT: Proton beam therapy

PET: Positron emission tomography

PTV: Planning target volume

RBE: Relative biological effectiveness

\section{Declarations}

\section{Ethics approval and consent to participate}

All procedures performed in studies involving human participants were in accordance with the ethical standards of the institutional research committee. For this type of study, formal consent is not required.

\section{Consent for publication}

Written informed consent was obtained from the patient for publication of this case report and any accompanying images.

\section{Availability of data and materials}

The data include individual patient data, but the data are available from the corresponding authors upon reasonable request.

\section{Competing interests}

The authors declare that they have no competing interests.

\section{Funding}

This case report was not supported by any funding.

\section{Authors' Contributions}

All listed authors contributed to the original manuscript. $\mathrm{YI}$ is the main radiation oncologist of this case and wrote the manuscript draft. MM and KJ coordinated and completed the manuscript. MS, HY, IS, MM, YT and YK supported proton beam therapy management. All authors have read and approved the manuscript of this case report. 
None

\section{References}

1. Bhansali MS, Fujita H, Kakegawa T, Yamana H, Ono T, Hikita S, et al. Pattern of recurrence after extended radical esophagectomy with three- field lymph node dissection for squamous cell carcinoma in the thoracic esophagus. World Journal of Surgery. 1997;21(3):275-81.

2. Hulscher JBF, Van Sandick JW, Tijssen JGP, Obertop H, Van Lanschot JJB. The recurrence pattern of esophageal carcinoma after transhiatal resection. Journal of the American College of Surgeons. 2000;191(2):143-8.

3. Liu Q, Cai XW, Wu B, Zhu ZF, Chen HQ, Fu XL. Patterns of failure after radical surgery among patients with thoracic esophageal squamous cell carcinoma: Implications for the clinical target volume design of postoperative radiotherapy. PLoS ONE. 2014;9(5):1-6.

4. Hsu PK, Wang BY, Huang CS, Wu YC, Hsu WH. Prognostic Factors for Post-recurrence Survival in Esophageal Squamous Cell Carcinoma Patients with Recurrence after Resection. Journal of Gastrointestinal Surgery. 2011;15(4):558-65.

5. Ku GY. Systemic therapy for esophageal cancer: Chemotherapy. Chinese Clinical Oncology. 2017;6(5):1-10.

6. Makishima H, Ishikawa H, Terunuma T, Hashimoto T, Yamanashi K, Sekiguchi T, et al. Comparison of adverse effects of proton and $\mathrm{X}$-ray chemoradiotherapy for esophageal cancer using an adaptive dose-volume histogram analysis. Journal of Radiation Research. 2014;56(3):568-76.

7. Hirano Y, Onozawa M, Hojo H, Motegi A, Zenda S, Hotta K, et al. Dosimetric comparison between proton beam therapy and photon radiation therapy for locally advanced esophageal squamous cell carcinoma. Radiation Oncology. 2018;13(1):1-9.

8. Williamson JF. Physics Contribution. Ini J Radiation Oncology Biol Phys. 1998;41(4):959-70.

9. Zhang X, Zhao K le, Guerrero TM, Mcguire SE, Yaremko B, Komaki R, et al. Four-Dimensional Computed Tomography-Based Treatment Planning for Intensity-Modulated Radiation Therapy and Proton Therapy for Distal Esophageal Cancer. International Journal of Radiation Oncology Biology Physics. 2008;72(1):278-87.

10. Gaitanidis A, Alevizakos M, Tsalikidis C, Tsaroucha A, Simopoulos C, Pitiakoudis M. Refusal of Cancer-Directed Surgery by Breast Cancer Patients: Risk Factors and Survival Outcomes. Clinical breast cancer. 2018 Aug;18(4):e469-76.

11. Nemoto K, Ariga H, Kakuto Y, Matsushita H, Takeda K, Takahashi C, et al. Radiation therapy for locoregionally recurrent esophageal cancer after surgery. Radiotherapy and Oncology. 2001;61(2):165-8.

12. Muroi $H$, Nakajima M, Satomura $H$, Takahashi M, Domeki Y, Murakami M, et al. Effectiveness of proton beam therapy on liver metastases of esophageal cancer: Report of a case. International Surgery. 2015;100(1):180-4. 
13. Ono T, Wada H, Ishikawa $H$, Tamamura $H$, Tokumaru S. Proton beam therapy is a safe and effective treatment in elderly patients with esophageal squamous cell carcinoma. Thoracic Cancer. 2020;11(8):2170-7.

14. Kato K, Tahara M, Hironaka S, Muro K, Takiuchi H, Hamamoto Y, et al. A phase II study of paclitaxel by weekly 1-h infusion for advanced or recurrent esophageal cancer in patients who had previously received platinum-based chemotherapy. Cancer chemotherapy and pharmacology. 2011 Jun;67(6):1265-72.

15. Jin J, Xu X, Wang F, Yan G, Liu J, Lu W, et al. Second-line combination chemotherapy with docetaxel and nedaplatin for Cisplatin-pretreated refractory metastatic/recurrent esophageal squamous cell carcinoma. Journal of thoracic oncology: official publication of the International Association for the Study of Lung Cancer. 2009 Aug;4(8):1017-21.

16. Ilson DH, Kelsen D, Shah M, Schwartz G, Levine DA, Boyd J, et al. A phase 2 trial of erlotinib in patients with previously treated squamous cell and adenocarcinoma of the esophagus. Cancer. 2011 Apr 1;117(7):1409-14.

17. Cho S-H, Chung I-J, Song S-Y, Yang D-H, Byun J-R, Kim Y-K, et al. Bi-weekly chemotherapy of paclitaxel and cisplatin in patients with metastatic or recurrent esophageal cancer. Journal of Korean medical science. 2005 Aug;20(4):618-23.

18. Lordick F, von Schilling C, Bernhard H, Hennig M, Bredenkamp R, Peschel C. Phase II trial of irinotecan plus docetaxel in cisplatin-pretreated relapsed or refractory oesophageal cancer. British journal of cancer. 2003 Aug 18;89(4):630-3.

19. Bidoli P, Stani SC, De Candis D, Cortinovis D, Parra HS, Bajetta E. Single-agent chemotherapy with vinorelbine for pretreated or metastatic squamous cell carcinoma of the esophagus. Tumori. 87(5):299-302.

20. Niibe Y, Hayakawa K. Oligometastases and oligo-recurrence: The new era of cancer therapy. Japanese Journal of Clinical Oncology. 2010;40(2):107-11.

21. Jingu K, Matsushita H, Takeda K, Umezawa R, Takahashi C, Sugawara T, et al. Long-term bresults of radiotherapy combined with nedaplatin and 5-fluorouracil for postoperative loco-regional recurrent esophageal cancer: Update on a phase II study. BMC Cancer. 2012;12.

22. Depypere L, Lerut T, Moons J, Coosemans W, Decker G, Van Veer H, et al. Isolated local recurrence or solitary solid organ metastasis after esophagectomy for cancer is not the end of the road. Diseases of the Esophagus. 2017;30(1):1-8.

23. Zhao X, Kang J, Zhao R. Abscopal effect of radiation on lymph node metastasis in esophageal carcinoma: A case report and literature review. Oncology letters. 2018 Sep;16(3):3555-60.

\section{Figures}




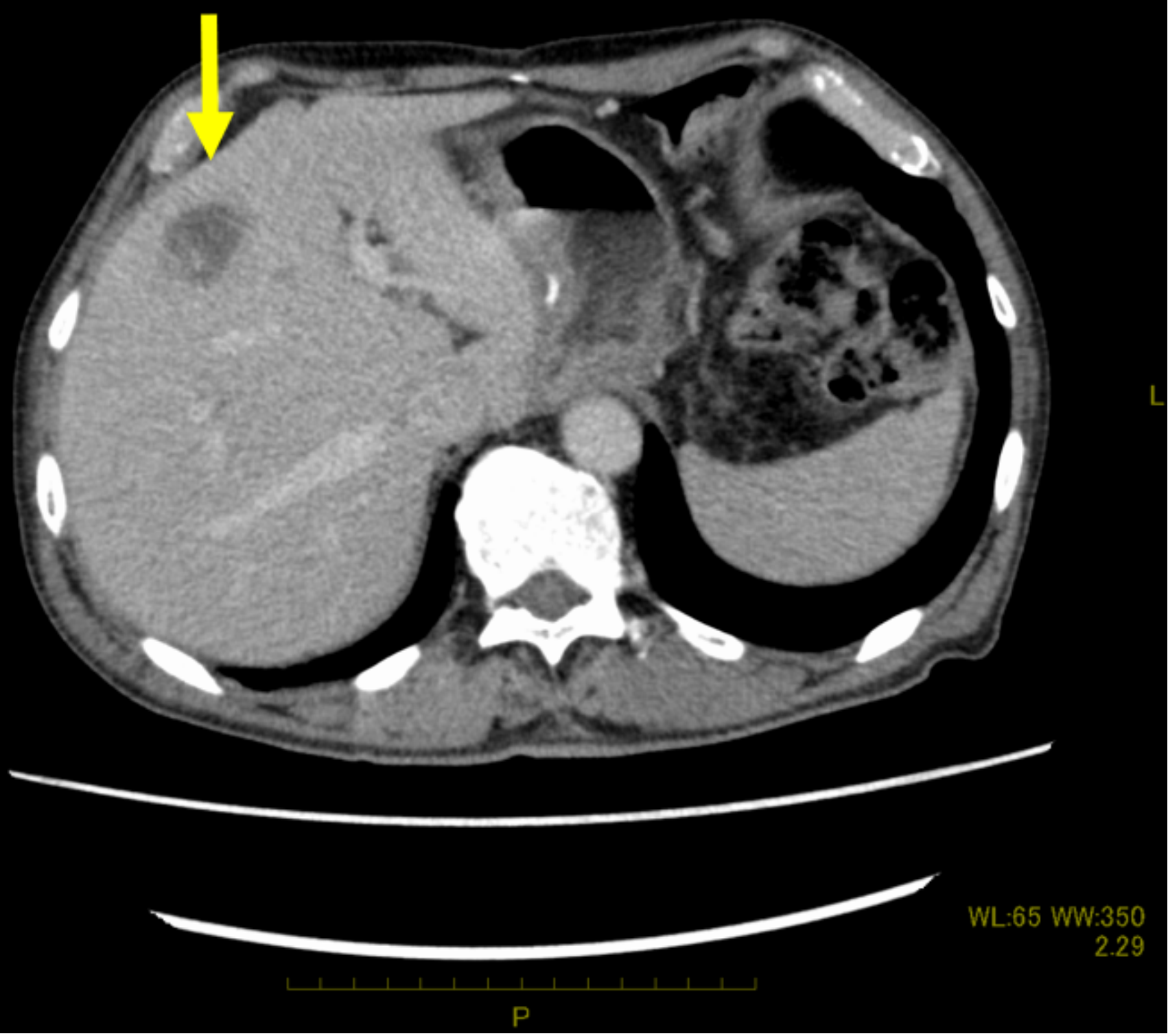

\section{Figure 1}

Axial enhanced computed tomography scan images of the abdomen showing a low-enhanced lesion of $21 \times 15 \mathrm{~mm}$ in size in the S4 area (yellow arrow). 

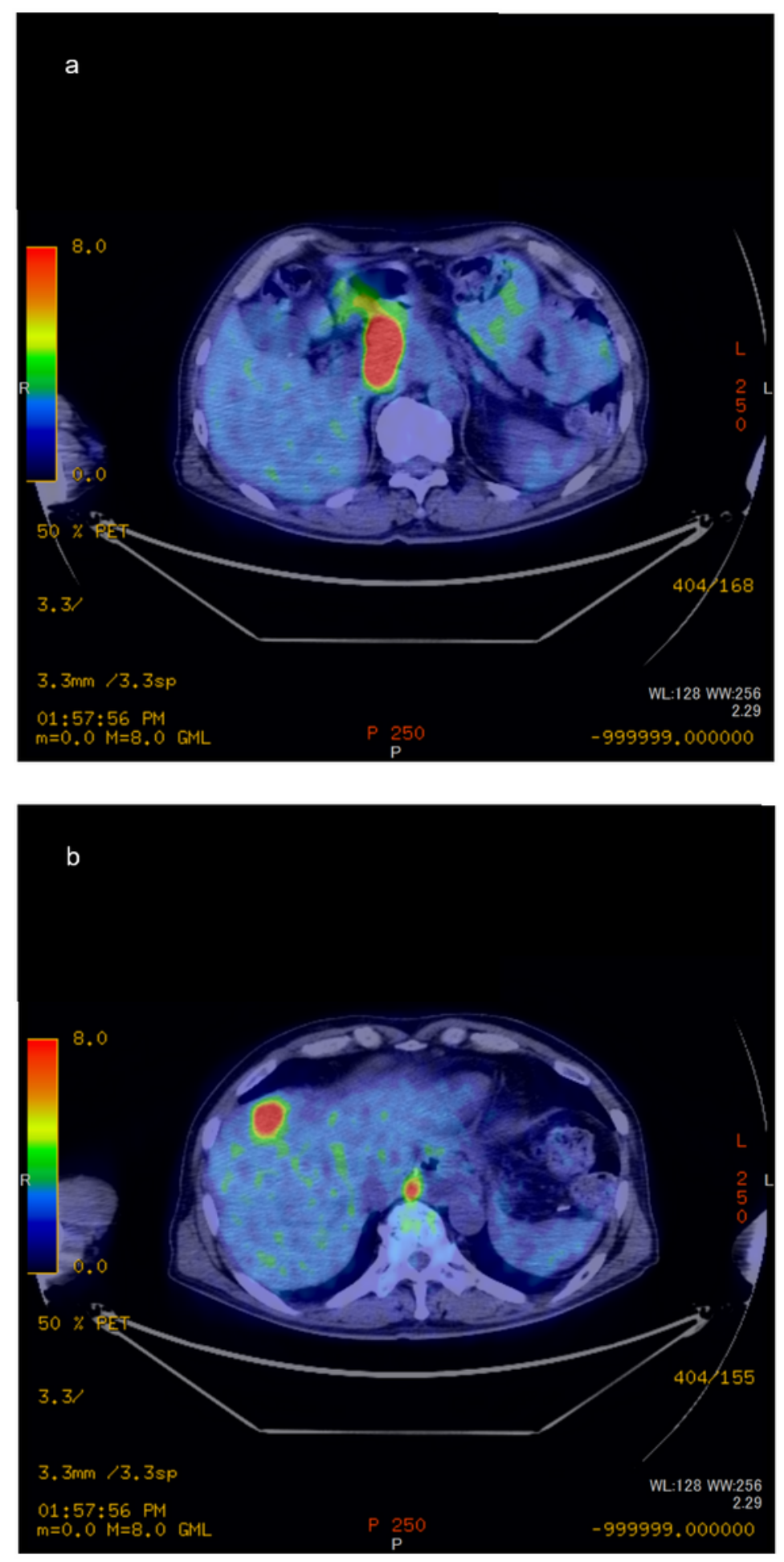

Figure 2

Positron emission tomography before proton beam therapy. Accumulation of fluorodeoxyglucose is shown in (a) para-aortic lymph node metastasis and (b) liver metastasis in the S4 area with para-aortic lymph node metastasis. 

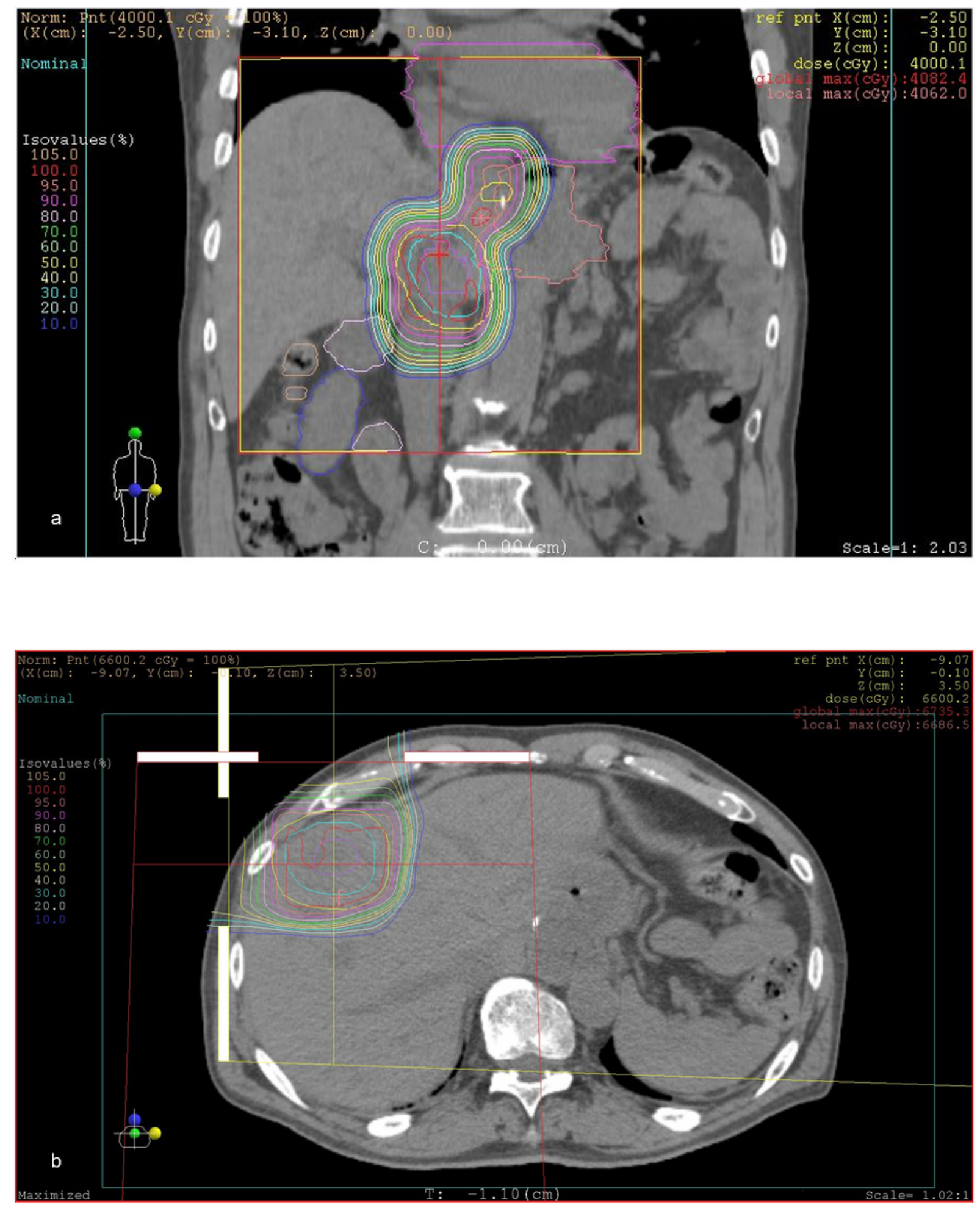

Figure 3

Dose distribution of proton beam therapy. (a) The lymph nodes were treated with 60 Gy relative biological effectiveness (RBE). The liver metastasis in S4 (b) was treated with 79.2 Gy RBE. 


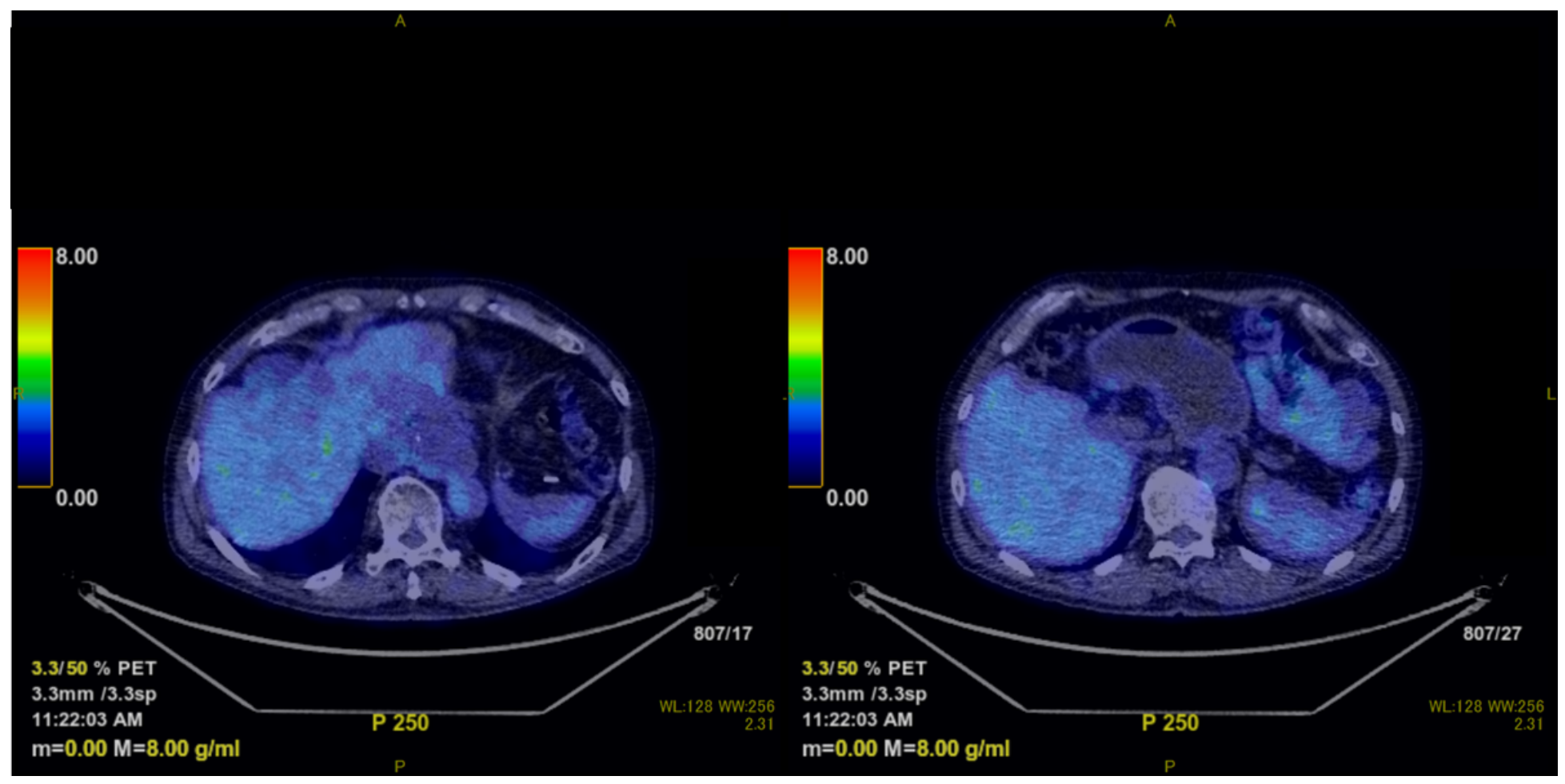

\section{Figure 4}

Positron emission tomography after proton beam therapy. Proton beam therapy resulted in the disappearance of fluorodeoxyglucose in all targets.

\section{Supplementary Files}

This is a list of supplementary files associated with this preprint. Click to download.

- CAREchecklistEnglish2013.pdf 